\title{
Nuevas adiciones a la flora del Perú, VIII
}

\section{Juan Montoya Quino', Eliana Linares Perea² \& Antonio Galán de Mera ${ }^{3}$}

${ }^{1}$ Herbario CPUN, Departamento de Biología, Universidad Nacional de Cajamarca, Cajamarca, Perú Estudios Fitogeográficos del Perú, Sánchez Cerro 219, Manuel Prado, Paucarpata, Arequipa, Perú `Laboratorio de Botánica, Facultad de Farmacia, Universidad CEU San Pablo, apartado 67, 28660 Boadilla del Monte, Madrid, España

\section{Correspondencia}

A. Galán de Mera

e-mail: agalmer@ceu.es

Recibido: 28 mayo 2019

Aceptado: 16 septiembre 2019

Publicado on-line: 26 septiembre 2019

Editado por: B. Cabezudo

\section{New additions to the flora of Peru, VIII}

Palabras clave: Biogeografía, flora, páramo, Perú.

Key words: Biogeography, flora, paramo, Peru.
Continuando con la serie de nuevos reportes para la flora peruana (Linares Perea et al., 2010, Montoya Quino et al., 2017a), damos a conocer cuatro nuevas citas con interés biogeográfico para el páramo del Departamento de Cajamarca. El páramo es una ecorregión situada sobre los 3000 $\mathrm{m}$ de altitud formada por pastizales acompañados de pequeños matorrales, caulirrósulas (Espeletia Mutis ex Bonpl.) y acantorrósulas (Puya Molina), y que se extiende desde Costa Rica hasta el $\mathrm{N}$ de la Cordillera Blanca (Perú) (Luteyn, 1999) y por los Andes orientales hasta Bolivia (Rangel, 2018). El clima se caracteriza por ser más cálido y húmedo que en el resto de los Andes. Las precipitaciones anuales son superiores a $1000 \mathrm{~mm}$ (Galán de Mera et al., 2015), con una variación estacional de temperaturas medias de entre $2^{\circ} \mathrm{C}$ y $10^{\circ} \mathrm{C}$, y 20-50 a $300 \mathrm{~mm}$ de precipitación mensual, cuyos mínimos se corresponden con los meses de junio a septiembre, con dos meses de aridez como máximo (Luteyn, 1999). El Departamento de Cajamarca contiene una flora muy rica con alrededor de 2700 especies registradas de las que unas 320 son endémicas (Hensold, 1999), lo cual es debido a la subsidencia paleozoica originada en el $\mathrm{N}$ del Perú que llevó a la formación de la depresión de Huancabamba, donde los Andes alcanzan la menor altitud $(2145 \mathrm{~m})$, separando los Andes del centro de los del norte y originando áreas montañosas aisladas y valles perpendiculares a la disposición general N-S de la cordillera (Reynel et al., 2013). Esta menor altitud de los Andes es además lo que propició mayores precipitaciones, que procedentes tanto del Océano Pacífico como de la Amazonía, dieron lugar a la presencia de páramo en el $\mathrm{N}$ de Perú.
Numerosas áreas del Departamento de Cajamarca no han sido aún bien estudiadas florísticamente, por lo que continuamente se vienen describiendo nuevas especies (Montoya Quino et al., 2017b) y detectando nuevas citas lejanas a sus centros de distribución (Weigend, 2004, Böhnert \& Weigend, 2016, Montoya Quino et al, 2017a).

Para comprobar estos nuevos registros se han tenido en cuenta la base de datos Tropicos (2019) y el material depositado en los herbarios B, BM, CPUN, COL, CUZ, F, FI, G, MA, MO, NY, US y USM, así como algunos trabajos monográficos (citados dentro de cada especie). Los especímenes estudiados se referencian en la figura 1 , cuyos mapas se prepararon con el programa QGIS 3.0.1 (QGIS Development Team, 2018).

Las nuevas citas que indicamos en esta ocasión pertenecen al piso bioclimático orotropical húmedo $\mathrm{y}$, desde el punto de vista biogeográfico, están dentro del sector Chota-Contumazá de la provincia Guayaquileña-Ecuatoriana (Galán de Mera et al., 2018):

Azorella corymbosa (Ruiz \& Pav.) Pers., Syn. PI. 1: 303 (1805) [Apiaceae]

PERÚ. Cajamarca: Cerro Gentiles, Mina Conga, 6 655'02.33"S-78²5'07.00"O, $3960 \mathrm{~m}$, 28/04/2012, J. Montoya, E. Linares \& A. Galán 4645 (CPUN, USP 3411).

Especie que se caracteriza por la formación de cojines duros y hojas trilobadas de $2-5 \mathrm{~mm}$ de longitud. Junto con $A$. pulvinata y $A$. compacta son las especies del género que forman los cojines más grandes, hasta de $1 \mathrm{~m}$ de altura. Se diferencia de $A$. pulvinata en que esta presenta las hojas 
pentalobadas de 2,5-3,5 $\mathrm{mm}$ de longitud; en $A$. compacta las hojas son enteras de 3-6 $\mathrm{mm}$ de longitud.

Habitat, distribución e interés biogeográfico: Forma cojines densos en áreas pedregosas del piso orotropical, en una altitud aproximada de 4000-4500 m, entre Colombia (Departamento de Caldas) y el centro de Perú (Departamento de Lima). Calviño et al. (2016) establecen las localidades más meridionales en el Departamento de Huánuco; sin embargo, Kahn et al. (2016), la llevan más al S, en el Departamento de Lima, y Mathias \& Constance (1976) indicaron su posible presencia en Bolivia. La nueva localidad parece ser la única conocida del páramo del $\mathrm{N}$ del Perú, por debajo del Chimborazo en Ecuador, indicando que a partir de nuestra nueva localidad hacia el $\mathrm{S}$ sigue su distribución por los Andes centrales y orientales peruanos como elemento de ambientes muy húmedos. Esto es coherente con la sugerencia de Cleef (1978) y de Rangel (2018) de llevar el páramo hasta los Andes orientales de Bolivia.

Material estudiado representativo: COLOMBIA. Caldas: Nevado Ruiz, alrededores del Nevado Ruiz y de Santa Isabel, $03^{\circ} 45^{\prime} 03.61^{\prime \prime} \mathrm{N}$ 74²4'59.84"O, 4140-4300 m, 10/10/1978, O. Rangel, H. Sturm \& O. Vargas 1820 (COL 221598). Cundinamarca: Macizo de Sumapaz, Alto de Caycedo, $04^{\circ} 08^{\prime} 05.00^{\prime \prime N}-74^{\circ} 14^{\prime} 32.00 " \mathrm{O}, \quad 3800-$ 3820 m, 09/01/1969, J. Cuatrecasas 27100 (COL 198430). Meta: Macizo de Sumapaz, Cerro Nevado, 035'08.00"N-74'06'35.00"O, 4100-4250 m, 06/07/1981, S. Díaz P., A.M. Cleef, O. Rangel \& S. Salamanca 2559 (COL 235159). PERÚ. Ancash: Pallasca, Cordillera de Pelagatos, 0809'19.32'S$77^{\circ} 47^{\prime} 57.29^{\prime \prime} O, 4450 \mathrm{~m}, 01 / 1920$, A. Weberbauer 7242 (F 549112). Huánuco: Pachitea, Tambo de Vaca, 0958'10.46"S-7603'06.89"O, 3900 m, 1024/06/1923, J. F. Macbride 4899 (F 535945).

Valeriana coarctata Ruiz \& Pav., Fl. Peruv. 1: 40, t. 68 (1798) [Caprifoliaceae]

PERÚ. Cajamarca: Cerro Gentiles, Mina Conga, 0655'00.07'S-78²5'05.48"O, 3980 m, 28/04/2012, J. Montoya, E. Linares \& A. Galán 4646 (CPUN, USP 3412).

Se trata de una de las especies del género con las hojas en roseta basal que caracterizan a la sección Sphaerophu Graebn. del subgénero Phyllactis (Pers.) Borsini (Graebner, 1906a; Eriksen, 1989a). Las flores en verticilastros capituloides, las hojas cilioladas, y las caulinares ternadas o cuaternas son su principal combinación de caracteres (Killip, 1937).

Habitat, distribución e interés biogeográfico: La hemos encontrado en fisuras anchas de roquedos formados por materiales volcánicos, al igual que en el resto de su areal (Galán de Mera et al., 2014). En todos los puntos de su distribución, entre La Paz (Bolivia) y Cajamarca (Perú), crece desde alrededor de los $4000 \mathrm{~m}$ de altitud hasta unos $5000 \mathrm{~m}$, siendo en este último departamento donde alcanza su localidad más septentrional. Esta nueva cita vuelve a indicar cómo en los pisos bioclimáticos superiores del $\mathrm{N}$ de Perú podemos encontrar una mayor cantidad de elementos florísticos del centro y $S$ de los Andes, mientras que en los basales predominan las especies comunes con Ecuador (Galán de Mera et al., 2015).
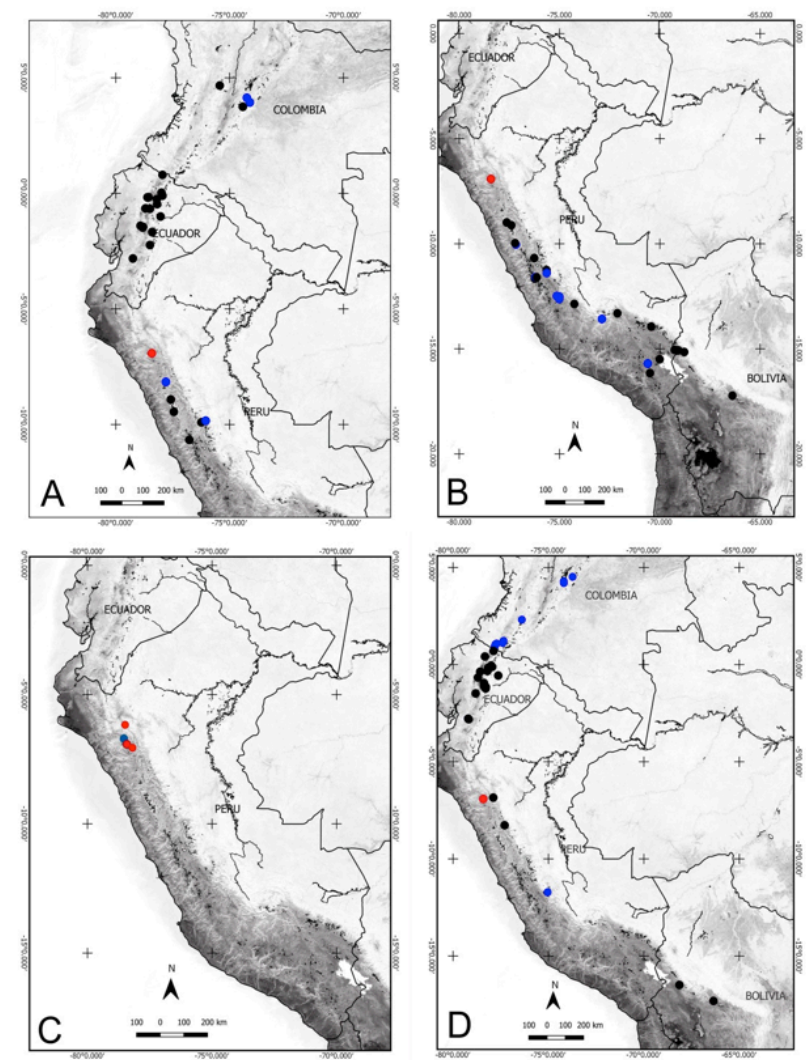

Figura 1. Mapas con la distribución conocida de las especies tratadas. A: Azorella corymbosa, B: Valeriana coarctata, C: V. hadros, D: Xenophyllum humile. Puntos azules- material de herbario estudiado, negros- referencias de bases de datos o bibliográficas, y rojos- nuevas citas.

Figure 1. Maps with the known distribution of the treated species. Blue dots - studied herbarium material, blacks - database or bibliographic references, and red- new records. 
Material estudiado representativo: PERÚ. Ancash: Cerca a Quicash, Aquia, $10^{\circ} 01^{\prime} 43.57^{\prime \prime}$ 77¹0'38.64"O, 15/05/1952, E. Cerrate 1594 (US 2270021). Apurimac: Abancay, Punas de Ampay, 1334'35.64"S- 7253'15.15"O, 3800 m, 06/1938, C. Vargas 1048 (CUZ 27376). Huancavelica: Conaica, $12^{\circ} 31^{\prime} 10.03^{\prime \prime} \mathrm{S}-7^{\circ} 00^{\prime} 28.22^{\prime \prime} \mathrm{O}, 4000 \mathrm{~m}$, 30/03/1952, O. Tovar 909 (US 2269997). Entre Conaica y Tinyajlla, $12^{\circ} 37^{\prime} 14.94^{\prime \prime} \mathrm{S}-75^{\circ} 00^{\prime} 54.73^{\prime \prime} \mathrm{O}$, 3800 m, 24/03/1952, O. Tovar 813 (US 2269995). Conaica, Huaytanayock-Tansiri cerca a Manta, 12²9'06.12"S- 75008'14.64"O, 4400-4500 m, 29/03/1953, O. Tovar 1132 (US 2269999). Junín:

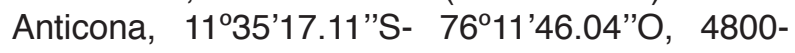
$4900 \mathrm{~m}, 21 / 12 / 1978$, M. Dillon \& B.L. Turner 1476 (F 1903184). Tarma, 11²3'08.16"S75³8'19.81"O, J. Pavón (BM 000947784). Puno: Santa Lucía, 1541'38.09"S-70³6'04.09"O, 4267 m, 20/02/1940, J.E. Sharpe 157 (US 1858931).

Valeriana hadros Graebn., Bot. Jahrb. Syst. 37: 443 (1906) [Caprifoliaceae]

PERÚ. Cajamarca: La Encañada, Kumulca, al Este carretera CajamarcaCelendín, $\quad 07^{\circ} 03^{\prime} 15.61^{\prime \prime S}-78^{\circ} 11^{\prime} 30.699^{\prime O}, \quad 3700$ m, 06/05/2001, I. Sánchez-Vega 10545 (CPUN 14656). Halgayoc, Caserío Coimolache, ladera Sur de la cuenca Las Flacas, 0609'59.11"S78²9'15.91'"O, 3300 m, 19/03/2008, J. CabanillasSoriano 1835 (CPUN 21592). Cerro Gentiles, Mina Conga, 0655'00.97'S-78 $25^{\prime} 04.07$ "O, $4000 \mathrm{~m}$, 28/04/2012, J. Montoya, E. Linares \& A. Galán 4647 (CPUN, USP 3413).

Endemismo del norte del Perú que se caracteriza por ser plantas perennes con un rizoma ramificado del que se desarrollan rosetas de hojas y varios tallos floridos ascendentes, prismáticos, pilosos, con verticilastros de dos brácteas opuestas sésiles, semejantes a las hojas; hojas pecioladas con láminas lanceoladas dentado-pinnatífidas, agudas en el ápice. El fruto es una cipsela con el cáliz persistente, cuya base soldada tiene una longitud de ca. 0,5 mm (Fig. 2).

Al parecer, el fruto se describe por primera vez, pues no figura ni en el protólogo de Graebner (1906b) ni en la descripción de Killip (1937). Por sus características morfológicas podemos deducir su pertenencia al grupo de $V$. pilosa (Eriksen, 1989b, Seminario-Cunya et al., 2016), dentro de la sección Sphaerophu Graebn.

Habitat, distribución e interés biogeográfico: La población encontrada presenta unos 11 ejemplares que viven en fisuras anchas de rocas volcánicas. El interés de estas citas es que son las primeras colectas tras su descripción a principios del siglo $X X$ en las cercanías de Hualgayoc (León, 2006), dentro de una macizo aislado geográficamente por el río Marañón al E, y la depresión de Huancabamba al $\mathrm{N}$.

Material estudiado representativo: PERÚ. Cajamarca: De Hualgayoc a Cajamarca, 06²'07.68'S-78³2'09.49"O, 4100-4200 m, 09/06/1904, A. Weberbauer 4228 (B 17844, G 00354485, USM 001013).

Xenophyllum humile (Kunth) V.A. Funk, Novon 7(3): 239 (1997) [Asteraceae]

PERÚ. Cajamarca: Cerro Gentiles, Mina Conga, 0654'59.83"S-78²5'04.92"O, $3987 \mathrm{~m}$, 28/04/2012, J. Montoya, E. Linares \& A. Galán 4648 (CPUN, USP 3414).

Se trata de la única especie peruana del género que presenta las hojas cilíndricas que caracterizan al grupo de Xenophyllum V.A. Funk propio del páramo de Colombia y Ecuador (Funk, 1997), lo que hace lógica esta nueva cita, situada en el páramo del norte de Perú.

Habitat, distribución e interés biogeográfico: Al igual que en el resto de las poblaciones conocidas, esta especie prefiere hábitats húmedos formando cojines en las cercanías de lagunas y suelos muy húmedos que se encuentran por encima de los $3700 \mathrm{~m}$ (Lauer et al., 2001). De las 14 especies conocidas de Xenophyllum en el Perú (Linares Perea et al., 2010; Beltrán, 2016), tal vez esta sea la que presenta una distribución menos conocida. Es muy abundante desde Colombia a Ecuador, con algunas localidades en el oriente peruano-boliviano que nos permiten aproximarnos a la idea de llevar la ecorregión del páramo hasta los Andes orientales de Bolivia. Aunque Funk (1997), sin pliego testigo, ya indicaba su presencia en el $\mathrm{N}$ de Perú, la población estudiada por Beltrán (2016) en el Departamento de Amazonas es de 1904.

Material estudiado representativo: COLOMBIA. Boyacá: Páramo de la Sarna, entre Sogamoso y Vado Hondo Valle Ancho, 05'40'24.93"N-72'48'44.89"O,3405m, 05/04/1973, J. Cuatrecasas (COL 238625). Cauca: Faldas del volcán Pucaré, $02^{\circ} 18^{\prime} 49.69^{\prime \prime N}-76^{\circ} 23^{\prime} 42.76$ "O, 4050-4150 m, 22/07/1976, R. Jaramillo-Mejía (COL 209784). Pucaré, entre Pilimbalá y el volcán Puracé, $\quad 02^{\circ} 22^{\prime} 00.00^{\prime \prime} \mathrm{N}-76^{\circ} 24^{\prime} 00.00^{\prime \prime O}$, 35004300 m, 14/11/2004, J.C. Betancur-Betancur 11215 (COL 511365). Cundinamarca: Bogotá, 
Nazaret, Santa Rosa, Parque Nacional Natural Sumapaz, $\quad 04^{\circ} 11^{\prime} 57.00^{\prime \prime} \mathrm{N}-74^{\circ} 11^{\prime} 41.00^{\prime \prime} \mathrm{O}, \quad 3370$ m, 02/10/1999, M. Ángel (COL 464191). Bogotá, Laguna de Chisaca, $04^{\circ} 16^{\prime} 58.00^{\prime \prime} \mathrm{N}-74^{\circ} 12^{\prime} 02.00^{\prime \prime} \mathrm{O}$, 3650-3700 m, 23/12/1959, J. Cuatrecasas 25746 (COL 85726). Bogotá, Localidad de Usme, el Carrizal, alrededores de la laguna el Pato, $04^{\circ} 18^{\prime} 35.00^{\prime \prime} \mathrm{N}-74^{\circ} 09^{\prime} 27.00^{\prime \prime} \mathrm{O}, 3703 \mathrm{~m}$, 17/01/2013, N. Castañeda \& A. Orejuela 1429 (COL 573371). Páramo de Chisaca, Laguna Verde, $\quad 05^{\circ} 12^{\prime} 56.10^{\prime \prime} \mathrm{N}-73^{\circ} 59^{\prime} 57.24^{\prime \prime O}, \quad 4000$ m, 16/09/1952, T. Van der Hammen 506 (COL 41819). Páramo de Chingaza, Fómeque, Laguna del Medio, 0430'30.26"N- 7344'36.39"O, 3100 m, 15/12/1963, G. Huertas 5831 (COL 161054). Fómeque, Lagunas de Buitrago, $04^{\circ} 45^{\prime} 28.00^{\prime \prime} \mathrm{N}$ $73^{\circ} 49$ 42.00"O, 12/08/2000, F. Zapata 66 (COL 466600). La Calera, Páramo de Chingaza, Laguna Seca, $04^{\circ} 31^{\prime} \quad 11.00^{\prime \prime} \mathrm{N}-73^{\circ} 44^{\prime} 13.00 " \mathrm{O}, \quad 3740$ m, 19/09/1972, R. Jaramillo-Mejía 5469 (COL 164956). Páramo de Chisaca, entre Las Lagunas y Nazareth, $04^{\circ} 10^{\prime} 19.64^{\prime \prime} \mathrm{N}-74^{\circ} 08^{\prime} 52.40^{\prime \prime} \mathrm{O}, 3510$

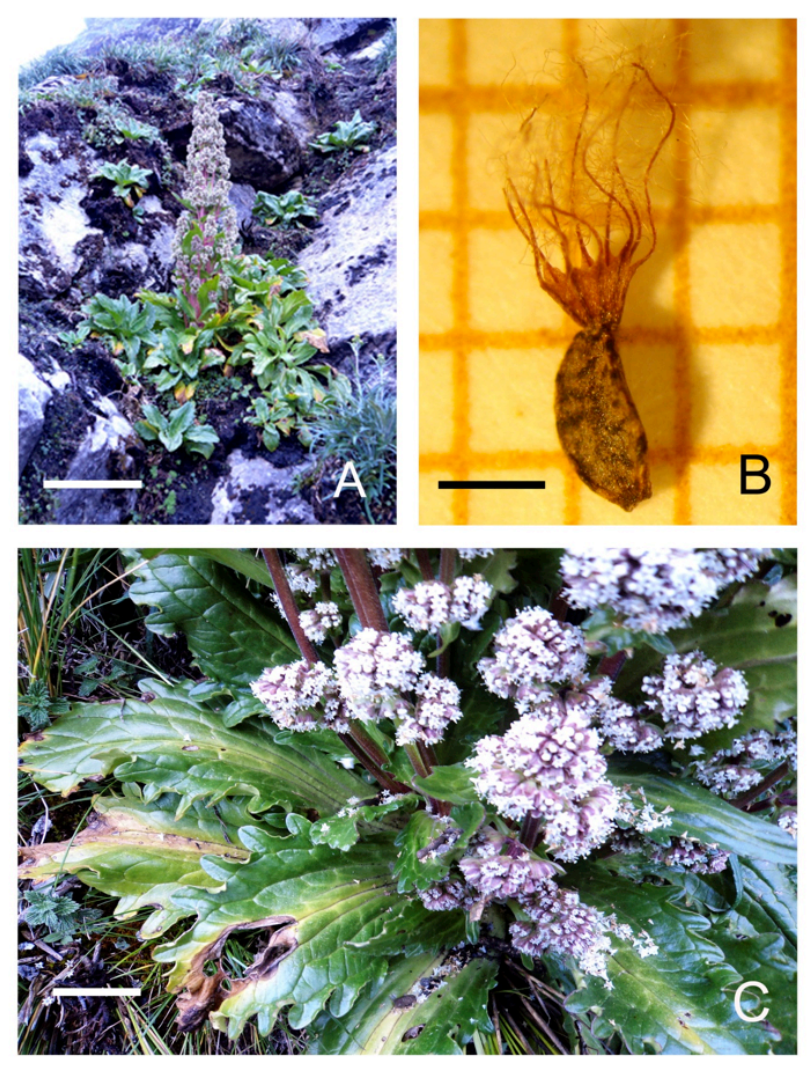

Figura 2. Valeriana hadros. A: ejemplares en su hábitat, B: fruto, C: detalle de las hojas basales (barra de A: 15 $\mathrm{cm}, \mathrm{B}: 1 \mathrm{~mm}, \mathrm{y} \mathrm{C}: 3 \mathrm{~cm})$.

Figure 2. Valeriana hadros. A: specimens in the wild, B: fruit, $\boldsymbol{C}$ : detail of the basal leaves (scale bar of $A: 15 \mathrm{~cm}$, $B: 1 \mathrm{~mm}$, and $C: 3 \mathrm{~cm}$ ). m, 25/08/1977, S. Díaz-Piedrahita 1131 (COL 174106). Páramo de Sumapaz, Chisaca, $1 \mathrm{Km}$ al S de Laguna Larga, $04^{\circ} 29^{\prime} 34.97^{\prime \prime} \mathrm{N}-73^{\circ} 43^{\prime} 41.47^{\prime \prime O}$, 3625 m, 25/08/1972, A.M. Cleef 5277 (COL 164955). Pasca, Páramo de Chisaca al sureste de la Laguna los Tunjos, 04¹7'24.00'N-74¹1'59.00"O, 3450 m, 07/11/1987, L.R. Sánchez 727 (COL 404686). Meta: Páramo de Sumapaz, cerro Nevado del Sumapaz, Superparamo Pico del Nevado, lado NE, $03^{\circ} 46^{\prime} 27.24^{\prime \prime} \mathrm{N}-74^{\circ} 24^{\prime} 47.81^{\prime \prime O}$, $4250 \mathrm{~m}, 11 / 01 / 1973$, A.M. Cleef (COL 238626). Nariño: Alrededores de Cumbal, $00^{\circ} 57^{\prime} 09.49^{\prime \prime} \mathrm{N}$ 77049'01.02"O, 3000-3600 m, 20/07/1952, R. Romero-Castañeda 3279 (COL 104091). Mallama, Chambú, $01^{\circ} 03^{\prime} 02.00^{\prime \prime} \mathrm{N}-77^{\circ} 46^{\prime} 43.00^{\prime \prime} \mathrm{O}, \quad 3318-$ 3739 m, 08/01/2009, L.M.Caballero 31 (COL 547807). Pasto, Volcán El Galeras, 01¹3'19.79"N77021'32.85"O, 3200-3500 m, 04/06/1946, R.E. Schultes 7955 (COL 60862). Sapuyes, volcán Azufral, $01^{\circ} 05^{\prime} 02.00^{\prime \prime} \mathrm{N}-77^{\circ} 43^{\prime} 08.00^{\prime \prime} \mathrm{O}, 4000 \mathrm{~m}$, 30/09/1979, L.E. Mora (COL 208465). Túquerres, entre Túquerres y volcán Azufral, $01^{\circ} 05^{\prime} 30.00^{\prime \prime} \mathrm{N}$ 77040'16.00"O, 3100-3900 m, 08/01/1952, A. Fernández-Pérez 1136 (COL204569). Yacuanquer, 0107'00.68'N- 77²3'59.23"O, 04/01/1943, M. Garganta 488 (COL 123261). ECUADOR. Azuay: Parque de las Cajas, 02 $48^{\prime} 44.81^{\prime \prime}$ 79'10'42.30"O, $4400 \mathrm{~m}, 23 / 10 / 1995$, V.A. Funk \& X. Montezuma 11432 (US 3321841). PERÚ. Junín:

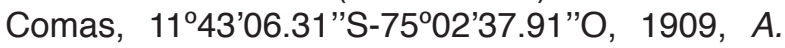
Weberbauer 6638 (USM).

\section{Bibliografía}

Beltrán, H. (2016). Sinopsis del género Xenophyllum (Asteraceae: Senecioneae) del Perú. Arnaldoa, 23(1), 351-362.

Böhnert, T. \& Weigend, M. (2016). Three new species records of Symplocos (Symplocaceae) from northern Peru. Revista peruana de biología, 23(3), 339-342. doi: http://dx.doi.org/10.15381/rpb.v23i3.12873

Calviño, C.I., Fernández, M. \& Martínez, S.G. (2016). Las especies de Azorella (Azorelloideae, Apiaceae) con distribución extra-argentina. Darwiniana, 4(1), 57-82. doi: http://dx.doi.org/10.14522/darwiniana.v4i1.681

Cleef, A.M. (1978). Characteristics of neotropical páramo vegetation and its sub-Antarctic relations. In C. Troll \& W. Lauer (Eds.), Geoecological relations between the southern temperate zone and the tropical mountains. Erdwissenschaftliche Forschung 11 (pp. 365-390). Wiesbaden: Franz Steiner.

Eriksen, B. (1989a). Notes on generic and infrageneric delimitation in the Valerianaceae. Nordic Journal of Botany, 9, 179-187. doi: https://doi. org/10.1111/j.1756-1051.1989.tb02113.x

Eriksen, B. (1989b). Valerianaceae. In G. Harling \& L. Andersson (Eds.), Flora of Ecuador No. 34 ( $\mathrm{pp}$. 3-60). Göteborg-Stockholm: University of Göteborg- 
Riksmuseum.

Funk, V.A. (1997). Xenophyllum, a New Andean Genus Extracted from Werneria s.l. (Compositae: Senecioneae). Novon, 7, 235-241.

Galán de Mera, A., Méndez, E., Linares Perea, E., Campos de la Cruz, J. \& Vicente Orellana, J.A. (2014). Las comunidades vegetales relacionadas con los procesos criogénicos en los Andes peruanos. Phytocoenologia, 44(1-2), 121-161. doi: 10.1127/0340269X/2014/0044-0576

Galán de Mera, A., Linares Perea, E. \& Montoya Quino, J. (2018). Mapa de vegetación de Cajamarca. Potencialidad de la vegetación para el uso de plantas medicinales. Cajamarca, PE: UPAGU-Municipalidad Provincial de Cajamarca-Yanacocha.

Galán de Mera, A., Sánchez Vega, I., Montoya Quino, J., Linares Perea, E., Campos de la Cruz, J., Vicente Orellana, J.A. (2015). La vegetación del norte del Perú: De los bosques a la jalca en Cajamarca. Acta Botanica Malacitana, 40, 157-190. doi: http://dx.doi. org/10.24310/abm.v40i0.2505

Graebner, P. (1906a). Die Gattungen der natürlichen Familie der Valerianaceae. Botanische Jahrbücher fur Systematik, Pflanzengeschichte und Pflanzengeographie, 37, 439-480.

Graebner, P. (1906b). Valerianaceae andinae. Botanische Jahrbücher fur Systematik, Pflanzengeschichte und Pflanzengeographie, 37, 436-451.

Hensold, N. (1999). Las angiospermas del Departamento de Cajamarca, Perú. Arnaldoa, 6, 141-184.

Kahn, F., Millán, B., Cano, A., La Torre, M.I., Baldeón, S., Beltrán, H., Trinidad, H., Castillo, S. \& Machahua, M. (2016). Contribución a la flora altoandina del distrito de Oyón, región Lima, Perú. Revista peruana de biología, 23(1), 67-72. doi: http://dx.doi.org/10.15381/ rpb.v23i1.11836

Killip, E.P. (1937). Valerianaceae L. Valerian Family. In J.F. Macbride (Ed.), Flora of Peru Vol. 13(2) (pp. 287321). Chicago, IL: Field Museum of Natural History.

Lauer, W., Rafiqpoor, M.D. \& Theisen, I. (2001). Physiographie, Vegetation und Syntaxonomie der Flora des Páramo de Papallacta (Ostkordillere Ecuador). Stuttgart: Franz Steiner.

León, B. (2006). Valerianaceae endémicas del Perú. Revista peruana de biología, 13(2), 663-668. doi: http://dx.doi.org/10.15381/rpb.v13i2.1929
Linares Perea, E., Campos de la Cruz, J., Nauray Huari, W., Vicente Orellana, J.A. \& Galán de Mera, A. (2010). Nuevas adiciones a la flora del Perú, V. Arnaldoa, 17(1), 99-112.

Luteyn, J.L. (1999). Páramos. A checklist of plant diversity, geographical distribution, and botanical literature. New York: The New York Botanical Garden.

Mathias, M.E. \& Constance, L. (1976). Umbelliferae. In G. Harling \& B. Sparre (Eds.), Flora of Ecuador No. 5 (pp. 1-72). Stockholm: Swedish Natural Science Research Council.

Montoya Quino, J., Torres Marquina, I., Linares Perea, E., Rodríguez Zegarra, C.C. \& Galán de Mera, A. (2017a). Nuevas adiciones a la flora del Perú, VII. Acta Botanica Malacitana, 42(2), 339-342. doi: http:// dx.doi.org/10.24310/abm.v42i2.2320

Montoya Quino, J., Linares Perea, E., Torres Marquina, I., Vicente Orellana, J.A. \& Galán de Mera, A. (2017b). Ageratina neblinensis (Asteraceae, Eupatorieae), una nueva especie para el norte del Perú. Gayana Botánica, 74(2), 288-292. doi: http://dx.doi.org/10.4067/S071766432017000200288

QGIS Development Team (2018). QGIS Geographic Information System. Open Source Geospatial Foundation Project. Beaverton, OR: OSGeo (https:// www.qgis.org/).

Rangel, J.O. (2018). Las plantas con flores de la región biogeográfica del Páramo (desde Costa Rica hasta Bolivia). In J.O. Rangel (Ed.), Colombia Diversidad Biótica Vol. XVI (pp. 1-82). Bogotá: Universidad Nacional de Colombia.

Reynel, C., Pennington, R.T. \& Särkinen, T. (2013). Cómo se formó la diversidad ecológica del Perú. Edinburgh: Royal Botanic Garden.

Seminario-Cunya, J.F., Rumay-Sánchez, L.D. \& Seminario-Cunya, A. (2016). Biología de Valeriana pilosa R. \&. P. (Valerianaceae): una especie en peligro de extinción de las altas montañas de Perú. Boletín Latinoamericano y del Caribe de Plantas Medicinales y Aromáticas, 15(5), 337-351.

Tropicos (2019). Tropicos. org. St. Louis, MO: Missouri Botanical Garden (http://www.tropicos.org).

Weigend, M. (2004). Additional observations on the biogeography of the Amotape-Huancabamba zone in Northern Peru: Defining the South-Eastern limits. Revista peruana de biología, 11(2), 127-134. 